Djuḥā ressuscité dans «Moha le fou Moha le sage » de Ben Jelloun

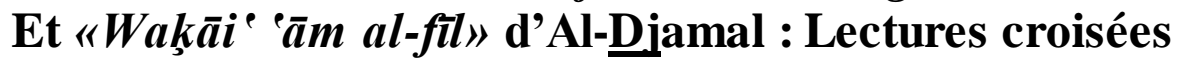

\title{
Zinat Chams
}

Département de Langue et de Littérature Françaises

Faculté des Lettres- Université de Hélouan

\section{Résumé}

zeinachams@yahoo.com

Cette recherche est consacrée à la résurrection du personnage de Djuhāā dans les deux littératures contemporaines francophone et arabe à travers deux œuvres ; la première en est «Moha le fou Moha le sage» de Ben Jelloun, auteur francophone d'origine marocaine, la deuxième, "Waķāie 'ām al-fîl» d'AlDjamal, journaliste et romancier égyptien. La problématique de cette étude se concentre sur l'impact du milieu social, culturel et politique sur la création des deux romans, impact qui se reflète dans l'intertexte ou l'arrière-texte qu'ils renferment. De même, cette recherche a analysé la divergence dans la réception de chacune des deux œuvres par les lecteurs, surtout en ce qui concerne l'assimilation de l'intertexte ou de l'arrière-texte qu'elle contient; cette analyse est basée sur la théorie du Lecteur Modèle d'Umberto Eco, tout en l'adaptant à l'héritage culturel de Djuhāa. Cette étude vise, en fin de compte, à démontrer la complémentarité entre la théorie d'Eco d'une part et les deux concepts de l'intertexte et de l'arrière-texte de l'autre part, et à souligner leurs importances dans la détermination du Lecteur cible de toute œuvre littéraire.

Mots-clés : Littérature comparée, Djuhāā, Intertextualité, Arrière-texte, Lecteur Modèle.

Peu de personnages, dans la tradition et la culture des peuples, ont connu un sort pareil à celui de Djuhāa, l'Humoriste oriental par excellence, l'auteur présumé d'un héritage anecdotique revendiqué par plusieurs communautés. Il est toujours vivant dans la tradition de ces sociétés, figurant aussi bien dans de courts textes oraux (anecdotes, proverbes...) que dans des ouvrages plus longs (romans, pièces de théâtre,etc). Ressusciter, dans des œuvres littéraires modernes, un tel personnage, n'est nullement gratuit ; il s'agit de jeter un regard scrutateur et espiègle sur la société contemporaine, vue à travers les yeux de ce héros mi-fou, mi-sage. C'est le prototype de l'homme du peuple armé uniquement de sa parole cinglante, qui tourne en dérision aussi bien les gouverneurs despotes et stupides que leurs sujets lâches et hypocrites. Aussi serait-il intéressant d'analyser la « mise en œuvre » de ce personnage bien riche dans deux ouvrages modernes, écrits dans deux langues différentes par deux auteurs orientaux de cultures bien distinctes.

\section{I- Corpus, problématique et plan de travail:}

L'objectif de cette recherche est d'analyser la reprise du mythe djuhaïen dans deux œuvres : Moha le fou Moha le sage » (1978), roman de l'écrivain 
marocain Tahar Ben Jalloun et "Waksäi' 'àm al-fil kama yarwìha al-shaykh

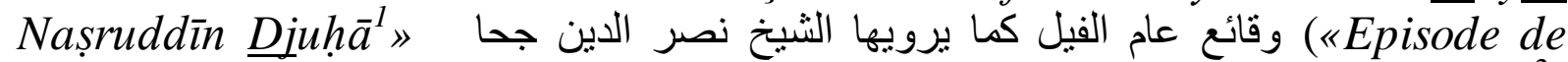
l'année de l'Eléphant d'après les Chroniques du Cheikh Naṣruddīn Djuhạa $»^{2}$ ) (1979), œuvre du journaliste égyptien Abdel-Fatāh Al-Djamal. Notre approche sera fondée, dans un premier temps, sur la mise en application de deux concepts de base pour toute étude visant à élucider la relation entre deux textes : l'intertexte et l'arrière-texte. Dans un second temps, notre réflexion portera sur le problème du lectorat que nous étudierons à la lumière du concept du Lecteur Modèle. Il nous a donc semblé primordial de commencer par éclaircir les concepts sur lesquels reposera ce travail.

Ainsi, selon Sophie RABAU, l'intertextualité, dans sa nouvelle acception, ne consiste plus à remonter aux sources des différents textes ou à élucider la relation qui pourrait exister entre eux. Elle va au-delà de cette conception restrictive. Selon ses dires, il faut repenser la littérature et l'envisager « comme un espace ou un réseau (...) où chaque texte transforme les autres qui le modifient en retour. »(Rabau, 2002: 15) Quant à l' « arrière-texte », il groupe, d'après la définition d'Alain TROUVÉ, tout ce qui a servi, de près ou de loin, à la création d'une œuvre ; cette notion implique la participation tant de l'auteur que celle du lecteur. L'auteur pourrait ainsi s'appuyer pour l'élaboration de son œuvre sur un ou plusieurs textes -oraux ou écrits-, un support visuel (peinture, objet d'art....), un simple souvenir, etc... ; le lecteur, lui, aurait pour charge de reconstruire, en cours de lecture, cet arrière-texte auctorial.(Trouvé, 2018:13)

Cependant, ce travail de déchiffrement ou de reconstruction n'est pas aisé pour tous les lecteurs dans la mesure où le lectorat n'est pas constitué d'un groupe homogène. Il dépend en grande partie de la culture du lecteur, de l'époque où il vit et du milieu auquel il appartient (qu'il soit francophone, arabophone ou autre). C'est cette diversité de types de lecteurs que nous tâcherons d'analyser en prenant en ligne de compte la théorie d'Umberto ECO. Selon ce sémioticien, chaque auteur choisit son «Lecteur Modèle »d'après les difficultés linguistiques, les références et les renvois qu'il insère dans son texte (Eco, 1985 :67, 68). Pour lui, chaque texte prévoit deux types de lecteurs : le «Lecteur sémantique » qui ne va pas au-delà du sens littéral du texte, et le "Lecteur critique » qui trouve un grand plaisir à en déchiffrer le sens implicite (Eco, $1992: 36$ ).

A cet égard, étant donné que notre corpus englobe un texte marocain francophone, il serait intéressant de souligner une particularité propre à toute production littéraire maghrébine d'expression française. Etudiant quelques problématiques des romans maghrébins, Charles BONN estime que ces ouvrages sont d'un abord assez difficile ; ils intéresseront plutôt des sociologues, des politiciens, ou en gros des chercheurs. (Bonn, 1986 : 68) Dans son article intéressant "Lecture et réception du texte maghrébin de langue française », Fouzia Bouguebina abonde dans ce sens, précisant que les textes maghrébins 
passionnent un type particulier de lecteur-chercheur qu'elle appelle le «lecteur critique universitaire »(Bouguebina, $2010: 3-8$ )

Pour plus de précision, nous classerons le Lecteur Modèle en trois catégories: le Lecteur sémantique (que nous désignerons, pour plus de commodité, par LS), celui qui lit le roman d'un jet et qui ne cherche, par sa lecture, que le plaisir de passer un bon moment ; le Lecteur critique (LC), plus cultivé, qui cherche à comprendre le dessous des cartes grâce à son propre bagage culturel sans pour autant aller au-delà des confins du texte lui-même ; et le Lecteur herméneute (ou Lecteur critique universitaire) (LH) qui n'hésite pas à faire des recherches, à confronter plusieurs textes pour saisir le sens du texte d'arrivée. A cette catégorisation, il serait pertinent d'ajouter une subdivision spatio-temporelle jusqu'alors négligée par les spécialistes: le Lecteur compatriote et contemporain de l'auteur, lecteur idéal de toute œuvre puisqu'il est ipso facto le destinataire premier du texte d'arrivée. Ainsi, en analysant notre corpus, nous étudierons dans quelle mesure un Lecteur sémantique compatriote de l'auteur (On l'appellera LComp) aussi bien qu'un Lecteur sémantique contemporain de la publication de l'œuvre (on l'appellera LCont) pourront reconstruire l'arrière-texte auctorial en lisant le texte d'arrivée. On étudiera aussi dans quels cas l'intertexte sera incompréhensible, voire imperceptible, pour un Lecteur sémantique non-contemporain (LNon-Cont) et/ou un Lecteur sémantique non-compatriote (LNon-Comp).

Notons aussi qu'un type insoupçonné de lecteur existe avant même la genèse du texte : c'est, en effet, l'auteur du texte d'arrivée. Celui-ci, pour écrire une œuvre qui renferme des traces intertextuelles, doit avoir déjà lu, vu ou entendu d'autres éléments, de nature textuelle ou non, pour les intégrer dans son œuvre sous forme d'intertextes ou d'arrière-textes. Ainsi l'auteur d'une œuvre intertextuelle est, par définition, le Lecteur Premier (ou plutôt Lecteur et/ou Récepteur Premier) d'un intertexte /arrière-texte qui sera à la base de son ouvrage. Dans cette recherche, nous commencerons par étudier comment le Lecteur/Récepteur Premier conçoit l'arrière-texte/intertexte djuhaïen en vue de construire le texte d'arrivée (ou la version finale de l'œuvre qui renferme l'ensemble de l'arrière-texte/intertexte conçu par l'auteur) ; celui-ci sera ensuite reçu par des Lecteur(s)/Récepteur(s) déjà cités.

Cette recherche comprend trois parties : la première est consacrée à l'étude de l'héritage djuhaïen tel que conçu par le Lecteur Premier de chacune des deux œuvres en question en vue de l'intégrer dans son roman. La deuxième partie portera sur l'analyse des avatars de ce patrimoine, transformé en arrièretexte/intertexte dans chacun des deux textes en question, tout en mettant l'accent sur la (ou les) catégorie(s) de Lecteur (s) capables(s) de l' assimiler.

II- Le Lecteur Premier et la résurrection de l'arrière-texte /intertexte djuhaien: 
Tout Lecteur premier qui tente de reprendre la tradition de Djuhā se trouve confronté à une gamme foisonnante d'anecdotes, de proverbes, de bribes d'Histoire relatant la biographie de personnes réelles insérés dans le folklore djuhāien, en plus d'un bon nombre d'œuvres littéraires mettant en scène le personnage de Djuhāa. Pour remonter aux sources de cet humoriste, disons que ce personnage - bien qu'originaire de la péninsule arabe- s'est reproduit dans la tradition d'autres pays tels que : l'Egypte, les pays du Maghreb, en plus du Nigeria, de Malte, de la Turquie, de l'Afghanistan, du Turkménistan, de la Russie, de la Chine,etc.....

( ( كاظم سعد الدين، ( . Nous aurons donc affaire à plusieurs «versions »de de Djuhāā dont nous retiendrons uniquement celles qui sont liées aux deux romans objet de notre étude, à savoir: les versions arabo-turque, égyptienne et maghrébine.

Le «modèle » arabe, la version la plus ancienne de cet humoriste, a vu le jour en Irak entre le VII ${ }^{\mathrm{e}}$ et le VIII ${ }^{\mathrm{e}}$ siècles de notre ère (Chams, 2002 :93). Les premiers ouvrages de compilation de ses anecdotes ont paru au $\mathrm{III}^{\mathrm{e}}$ siècle de

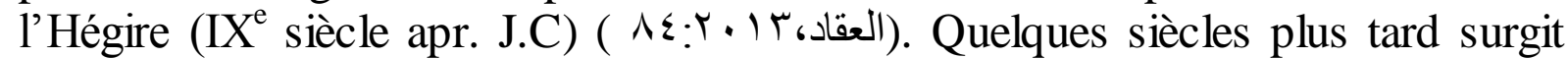
son homologue turc Al-Khudja Nașruddīn; C'est le "Djuhā turc », tel que le nomme Dr An-Nadjār, spécialiste de la littérature populaire arabe. Selon la même source, cet anecdotier serait un contemporain de Tamerlan ou Tīmūr

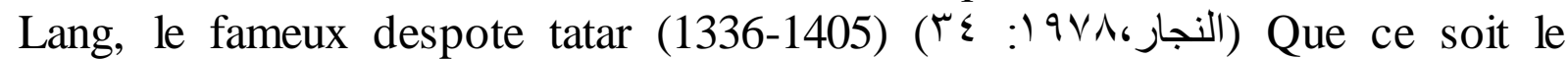
modèle arabe, turc, ou tout autre modèle postérieur, cet humoriste garde souvent les mêmes caractéristiques qu'Albert Memmi a résumées en ces termes : «Jeha (Djuhāa) est un personnage comique, involontairement en quelque sorte, parce qu'il est pauvre, démuni et maladroit ; mais volontairement aussi :il est taquin, fainéant, rusé, voleur...(....) Il fait l'idiot, mais pour se défendre. (...)Le personnage de Jeha est toujours un homme du peuple. Comme les gens du peuple, il ne dispose pour se défendre que des armes de la ruse et de l'esquive » (Memmi, 1991 : 57).

Quant au modèle égyptien de cet humoriste, selon les dires de Dr AnNadjār, il a fait son apparition en Egypte depuis l'ère islamique, surtout dans les périodes de troubles, telles que l'ère des Tulunides et des Ikhchidides, et a atteint son apogée sous l'Empire Ottoman. Selon la même source, c'est le modèle égyptien qui se répandra par la suite non seulement dans le monde arabe

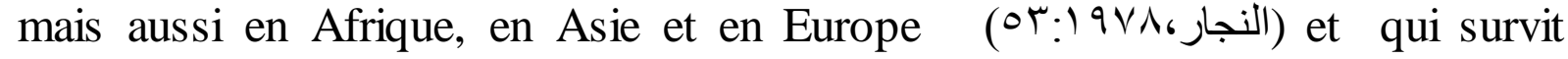
jusqu'à nos jours dans la Vallée du Nil. Il devient le type même de l'égyptien "fahlawi » : un débrouillard, coupeur de cheveux en quatre, ergoteur si besoin

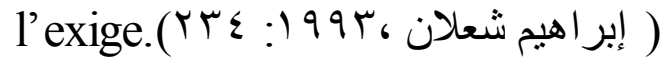

En ce qui concerne la reprise du modèle djuhāien au Maghreb, notons que Bernard Chanfrault, spécialiste du folklore arabe, estime que Djuhā incarne le "tunisien moyen» qui essaie d'allier la culture tunisienne traditionnelle et la technologie occidentale qui souvent le dépasse. (Chanfrault, 1991: 15) Il est bien 
intéressant à noter que la tradition de Djuḥā, dans toutes les cultures déjà citées, est formée d'un héritage hybride : anecdotes transmises oralement ou recueillies dans des œuvres , proverbes, caricatures, bandes dessinées, œuvres littéraires,.... Aussi la notion d'arrière-texte correspond-elle mieux que celle d'intertexte pour qualifier cette riche production accumulée à travers des siècles dans des cultures les plus variées.

Une question s'impose : quoi de neuf sur Djuhā pour qu'il soit repris par deux écrivains « sérieux » du $\mathrm{XX}^{\mathrm{e}}$ siècle ? La réponse nous est livrée par Al'Aķād, dans un ouvrage entièrement consacré à cet humoriste. Selon ce penseur, l'humour lié à ce personnage peut être défini, entre autres, comme "humour propre aux périodes de transition »: c'est un type d'humour inventé «à propos», pendant des périodes de décadence lorsqu'un créateur "génial et inspiré » recourt à « un personnage imaginaire déjà existant » pour en faire un prototype d' "hypocrisie, d'affectation et des traditions creuses.» ( العقاد، 1 ( + V $V)^{3}$ Rien donc d'étonnant à ce que les deux auteurs, Ben Jelloun et Al-Djamal, fassent de Djuhāa le personnage principal de deux romans évoquant respectivement deux sociétés orientales modernes en pleine mutation: le Maghreb et l'Egypte. Il s'avère donc que Djuhāa est une figure de proue des périodes de transition, d'autant plus que la parution des deux œuvres en question coïncide avec d'importants événements sur le plan sociopolitique dans les deux pays. La Vallée du Nil, après le troisième conflit arabo-israélien en 1973, était la scène d'importants troubles sociaux, tels les émeutes de 1977, la montée des courants islamistes et une certaine dérive autoritaire sur le plan politique. La situation économique s'est détériorée à cause de l'inflation et des dettes extérieures qui ne cessaient d'augmenter. (جلال أمين، 199 (ج) Sur le même côté de la Méditerranée, le Maroc s'est trouvé confronté presque aux mêmes enjeux : les émeutes de Casablanca en 1965, suivies de la proclamation de l'état d'exception jusqu'en 1970. Les deux ans suivants ont témoigné d'un coup d'État avorté (1971) et d'une tentative d'assassiner le roi (1972), qui ont mené, en fin de compte, à la modification de la Constitution. Le Maroc a également souffert de grands problèmes frontaliers avec l'Algérie. (صلاح العقاد، (199) (19)

C'estau sein de ces deux sociétés que Djuhāa a été ressuscité dans les deux ouvrages cités. Nous allons donc étudier, dans chaque roman, l'arrièretexte /intertexte lié à cet humoriste, et sa réception par les différents lecteurs potentiels. Nous commencerons par le personnage du roman arabe, plus proche de son ancêtre folklorique, ensuite nous analyserons le héros du texte francophone, fruit d'une double culture orientale et occidentale, qui prend ses distances avec le Djuḥā traditionnel.

\section{A-Djuhāa dans "Waksāi ' $\bar{a} m$ al-fïl» :}

Cette œuvre d'Al-Djamal met en scène un héros qui reproduit à plusieurs égards la version «arabo-égyptienne » de Djuhā. Notons d'abord que cet opuscule formé d'une soixantaine de pages compile une trentaine d'anecdotes et 
certains proverbes attribués à Djuhā dans les traditions égyptiennes. Dans l'exemple suivant, un passage $\overline{\mathrm{du}}$ texte d'arrivée groupe deux intertextes djuhaïens différents dont l'un est habilement emboîté dans l'autre.

$$
\text { جاءت الوفود جحا مستجيرين، قالوا: }
$$

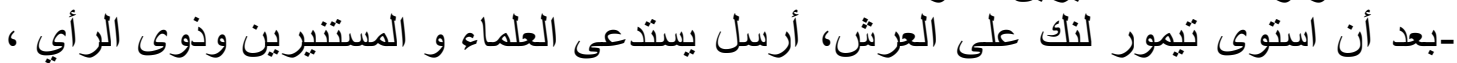

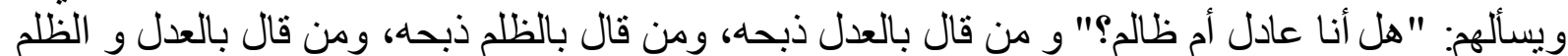

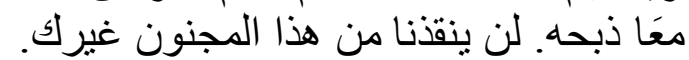
ـ ليكن في علمكم إنى إن استجبت لكم قامرت مع هذا السفاح المجنون بر أسى(....)

$$
\text { -فاضى واتر عشى جيالك. }
$$

وبكل حرص وحيطة، جاء بحماره إلى مقر السلطان.(....) سأله السلطان:

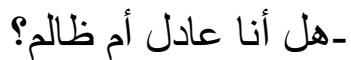

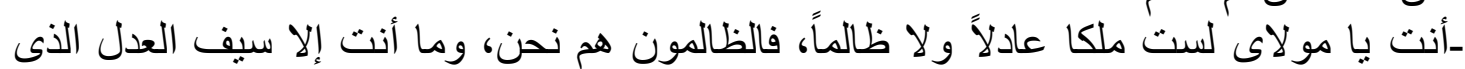

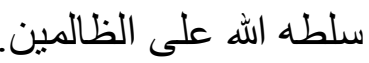

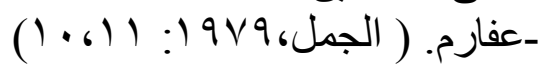

Des délégations (de villageois) viennent implorer le secours de Djuhāa ; ils lui disent: Tamerlan, ayant tenu les rênes du pouvoir, a donné l'ordre aux savants et aux gens d'esprit de comparaître devant lui pour qu'ils répondent à la question suivante: "Suis-je juste ou injuste?»Ceux qui répondent par «juste» sont égorgés, ceux qui disent : «injuste» sont égorgés et ceux qui répondent par "juste et injuste en même temps » sont égorgés. Personne d'autre que toi ne peut nous sauver.

-Sachez-le bien : si je satisfais à votre demande, je risquerai d'avoir la tête coupée à cause de ce fol assassin.

- Ô Dju hăa, (fais gaffe), la fièvre te rongera.

- Je ne sais quoi faire de ma peau,j'en tremblerai.

Avec toutes les précautions possibles, il amena son âne au palais du Sultan. Celui-ci lui demanda :

- Suis-je juste ou injuste?

- Mon Seigneur, vous n'êtes ni un monarque juste ni injuste, c'est nous qui sommes les injustes, vous n'êtes que le sabre de la justice qu'Allah envoya pour châtier les injustes.

- A la bonne heure!

Ce passage est un exemple bien réussi de la «contamination » qui, selon la terminologie genettienne, est un "mélange à doses variables» de deux intertextes (Genette,1982:303). En effet, ce texte reproduit presque fidèlement

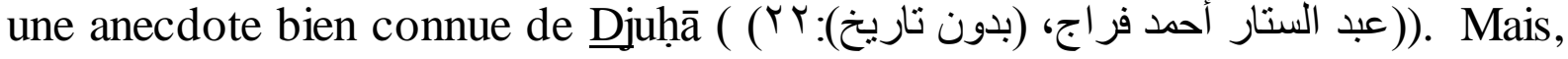
au milieu de cet intertexte, reproduit en arabe classique, on note la présence d'un 
court proverbe en dialecte égyptien, avec une certaine déformation ; en voici la traduction : "On dit à Djuhăa : Ton chien a de la fièvre; il répond: (T'en fais

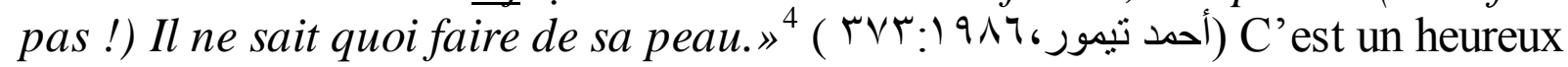
choix, puisque le proverbe joue ici le rôle d'un prélude -ou une anticipation- au violent face-à-face qui aura lieu entre Djuhāa et Tamerlan. Pourtant, il faut souligner que l'insertion de ce proverbe se fait imperceptiblement dans le texte d'Al-Djamal : rien n'indique qu'il s'agit ici d'un proverbe, d'autant plus que c'est un proverbe peu connu par les Egyptiens eux-mêmes. Dans ce cas, quelques LComp - seulement seraient à même de déceler qu'il s'agit là d'un intertexte.

Or, d'autres arrière-textes/intertextes sont plus subtils à déceler. Par exemple, le roman s'ouvre sur un dialogue entre les deux colombes, narratricescommentatrices de l'action, qui discutent de «l'étymologie»du nom propre « Djuhạa », prétexte ingénieux pour introduire ce héros au lecteur.

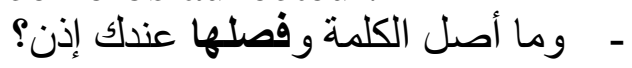

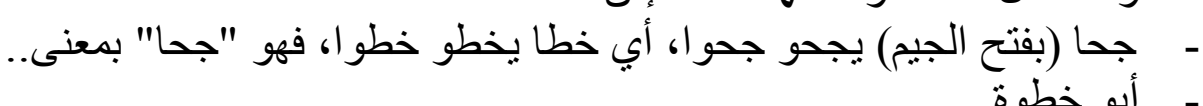

- ـ - تمام، أبو خطوة في المكان، بخفة القدم و اليد و اللسان. بمعنى.....

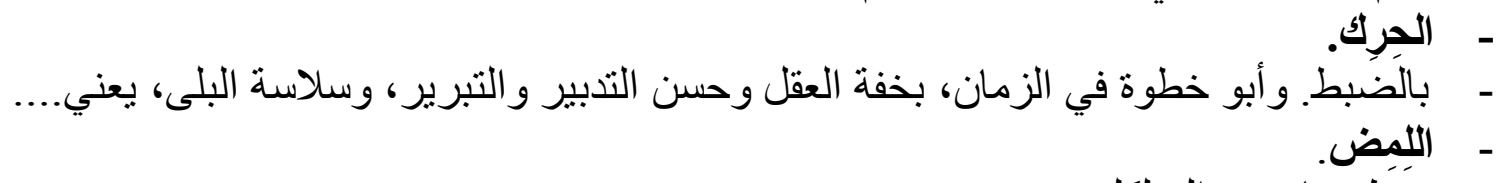

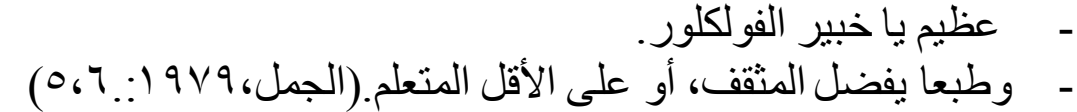

- Peux-tu me dire quelle est, pile-poil, l'origine de ce mot?

- $\quad$ Djuhā : nom commun et adjectif, désigne une personne qui fait de grands pas, c'est-à-dire ......

- $\quad$ Abū Khatwa (littéralement :faiseur de grands pas)

- Exactement, Abū Khatwa qui franchit l'espace grâce à l'agilité de ses jambes, de ses mains et de sa langue, c'est-à-dire...

- Un débrouillard.

- $\quad$ Parfait. Et aussi, Abū Khatwa qui franchit le temps grâce à son habilité à gamberger et argumenter, c'est-à-dire ...

- Un coupeur de cheveux en quatre.

- $\quad$ Super, mais t'es un spécialiste du folklore!

- Certainement, il est préférable que ce soit un homme cultivé, ou du moins éduqué. » 
D'après ce dialogue, « D «Abū Khatwa»; cette expression, qui constitue un arrière-texte puisé des traditions populaires égyptiennes, signifie "toute personne capable de franchir

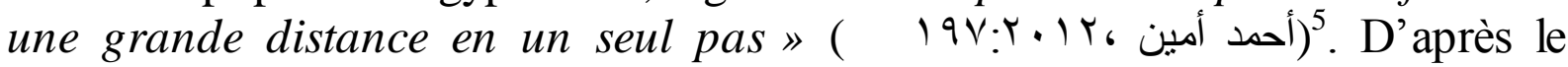
dialogue des deux colombes, Djuhāā est doublement «Abū Khatwa», étant donné qu'il est un « débrouillard » et un maître en l'art d'argumenter.

Conjointement à cet arrière-texte, le passage précité est un exemple d'un entrelacement de différents intertextes. Le premier résulte de l'insertion, dans ce passage écrit en langue arabe soutenue, de mots appartenant à l'idiolecte égyptien, qui, selon la définition genettienne, est l'ensemble de «locution(s) propre(s) ...à une langue ou un état de langue »(Genette, 1982 :

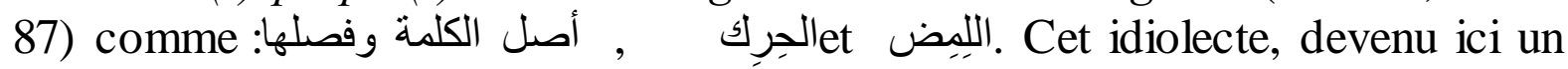
«texte dans le texte» ou intertexte, a pour effet d'intégrer le personnage folklorique de Djuhā dans la société égyptienne moderne. En effet, l'emploi de l'idiolecte égyptien parsemant un passage rédigé en langue classique n'est pas sans effet sur «l'univers spatio-temporel» ou la «diégèse » du roman (Genette,1982 : 341). On assiste donc à une «transdiégétisation» dans laquelle "une action peut être transposée d'une diégèse dans une autre, par exemple d'une époque à une autre, ou d'un lieu à un autre, ou les deux à la fois » (Genette, 1982 : 343) Ainsi le Djuhā d'Al-Djamal, qui vivait pendant "l'année de l'Eléphant » sous le règne de Tamerlan, devient-il, grâce à cet intertexte, un citoyen égyptien moderne. Le but de cette transdiégétisation est donc la "proximisation » qui vise à "rapprocher (la diégèse du texte) et l'actualiser aux yeux de son propre public.» (Genette, 1982 :351) Or, ce même procédé qui rend le texte plus proche du LComp, le rend assez obscur pour tout LNoncomp.

Bien plus, ce passage comporte aussi un arrière-texte camouflé sous la forme d'un intertexte bien commun : la citation. Pour remonter à l'étymologie du nom « Djuhāa », la colombe fait semblant de citer un article de dictionnaire :

$$
\text { - - }
$$

- Djuhāa : nom commun et adjectif, désigne une personne qui fait de grands pas.

Or, il existe déjà dans les dictionnaires arabes un article dont l'entrée est effectivement le radical arabe جحا is en voici l'énoncé :

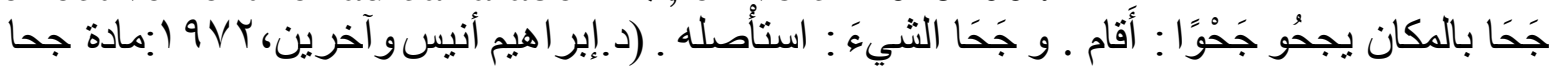

Selon la colombe, le radical arabe جَ signifie «faire un pas», alors que, d'après les dictionnaires arabes, le vrai sens en est "résider en un lieu » ou "extirper quelque chose». Il s'agit donc d'un article de dictionnaire presque créé de toutes pièces. On ne peut donc parler ici d'une citation proprement dite, car cet énoncé est apocryphe. Puisqu'il s'agit tout simplement 
d'imiter le « style » d'un dictionnaire, on pourrait donc parler d'un arrière-texte sous une forme bien usitée de l'intertexte.

Mais ces différents arrière-textes et intertextes sont-ils pareillement perçus par les différents lecteurs de cet ouvrage ? En lisant ce passage, un LS saisira en gros la signification du texte (l'ébauche du caractère de Djuhāa) sans chercher à aller plus loin. De son côté, un LC notera l'humour contenu dans cet énoncé. Il ne manquera sans doute pas de remarquer que la forme du texte lui rappelle celle d'un article de dictionnaire et de saisir la proximisation dès les premières lignes du roman. En ce qui concerne le LH, poussant plus loin son désir de compréhension globale, il effectuera une courte recherche sur l'article جحا dans un dictionnaire; il sera ainsi le seul lecteur capable de saisir pleinement l'humour contenue dans l'énoncé. Notons aussi, à cet égard, l'effet produit par l'insertion dans le texte d'arrivée de quelques mots de l'idiolecte égyptien provenant de la langue turque (que nous appellerons tout court: idiolecte turco-égyptien), surtout lorsqu'il s'agit d'un intertexte assez ambigu qui clôt le roman et détermine sa fin aussi bien que sa finalité. Mais, pour mieux saisir la portée de cet intertexte, on devrait d'abord passer en revue les événements qui l'ont précédé : l'éléphant, dont le roi a «doté » le village de Djuhāa, y a fait un grand ravage. Sous l'instigation des villageois, le héros vient rencontrer Tīmūr Lang dans le but de lui demander de les délivrer de ce « cadeau ». Or, dès qu'il commence à évoquer l'animal chéri du despote, celuici devient enragé, menace de tuer toute personne osant inquiéter son cher éléphant. Pour sauver sa peau, Djuhāa fait volte-face et dit au roi qu'il vient simplement lui demander..... de donner l'ordre d'emmener au village une femelle pour dissiper la solitude de son animal chéri. Djuhāa a donc été récompensé, alors que le village a été complètement détruit à cause de la gloutonnerie des deux éléphants. Tel est le dernier épisode du roman, suivi d'un court texte assez obscur, voire désinvolte, qui termine le roman :

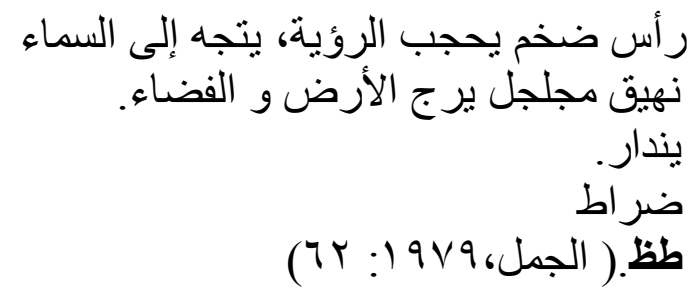

"Une grosse tête bloque la vue et se dirige vers le ciel

Un braiement sonore retentit entre la terre et le ciel.Il se retourne. Une pétarade est lâchée.Je m'en fous. "

Le dernier mot du roman طظ « d'une part, parce qu'il fait partie de l'idiolecte égyptien moderne au sein d'un texte rédigé en arabe classique, et, d'autre part, parce qu'il est à l'origine emprunté à la langue turque classique et exprime une indifférence dédaigneuse ${ }^{6}$. Mais comment les différents lecteurs peuvent-ils interpréter ce fameux « $\boldsymbol{J} \boldsymbol{e}$ m'en fous »? Le LS se contentera d'en rire, alors que pour les LC et LH, cette 
expression soulèvera plus d'une interrogation. Qui est le véritable locuteur de cet énoncé ? Et quelle en est la signification ?

Le locuteur pourrait bien être Djuhạa , puisqu'il est question de son âne dans le passage (notons que l'âne, dans ce roman, ne parle jamais ; il n'est pas un locuteur).L'énoncé final, ajouté à l'acte désinvolte qui l'a précédé (la pétarade lâchée), reflèteront une indifférence affichée aux malheurs survenus au village, malheurs dont Djuhāa est indirectement la cause. Mais si les deux lecteurs, allant plus loin, pourront attribuer ce même énoncé problématique au narrateur luimême, celui-ci visera donc à rappeler au lecteur qu'il s'agit d'un simple conte, laissant ainsi passer impunément la critique sociale que le texte renferme. De telles hypothèses inquièteront les $\mathrm{LC}$ et $\mathrm{LH}$; ils chercheront le sens implicite de cet arrière-texte qui, par sa place à la fin du roman, semble acquérir une certaine signification bien particulière.

Pourtant, le Djuhāa d'Al-Djamal se rapproche du LCont surtout grâce à certains "anachronismes » ou " détails stylistiques et thématiques modernes » qui «émaille(nt) une action moderne ». Grâce à cette "dissonance ponctuelle par rapport à la tonalité d'ensemble de l'action », l'anachronisme remplit diverses fonctions auprès du lecteur : "il frappe, surprend, amuse ou donne à penser. »(Genette, 1982: 358) Certains anachronismes reflètent directement et sans ambages la réalité égyptienne des années soixante-dix. Ainsi, vers la fin du roman, les villageois, exaspérés des ravages que fait l'éléphant de Tamerlan dans leur terre, exhortent Djuhāà à aller rencontrer le roi pour lui demander d'évacuer l'éléphant du village. La veille de sa rencontre avec Tamerlan, Djuhāā a fait un rêve étrange : il s'est vu entouré de Munkar et Nakīr -les deux anges responsables d'interroger les morts selon la tradition islamique- ; ceux-ci lui ont fait subir un interrogatoire bien qu'il ne soit pas encore mort. Étonné, Djuhāa s'est écrié :

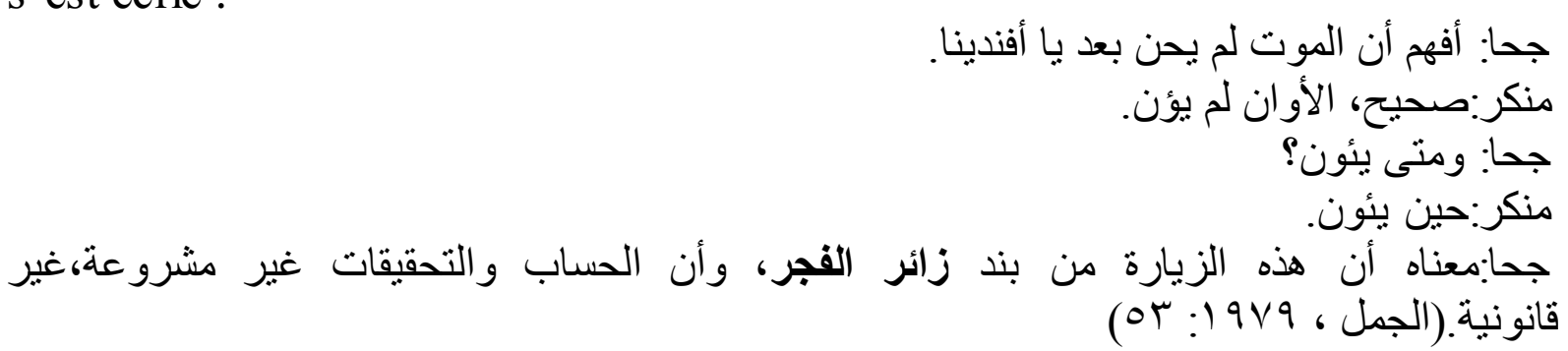

Diuhāa : Votre majesté, à ma connaissance, ma dernière heure n'a pas encore sonné.

Munkar : C'est vrai, elle n'a pas sonné.

Diu $\bar{a} \bar{a}:$ Et quand sonnera-t-elle.?

$\bar{M}$ unkar : Lorsqu'elle sonnera.

Djuhä : Il semble que ce soit une des visites effectuées par les «visiteurs de l'aube ». Cet interrogatoire et cette enquête sont donc illégitimes, illégaux. » Dans ce passage, Djuhāa fait allusion à l'Egypte des années soixante et soixantedix, grâce à l'anachronisme qui réside dans l'emploi de l'expression "visiteurs 
de l'aube ». A cette époque, elle désignait certains membres des services de sécurité qui avaient l'habitude d' « effectuer des visites à l'aube » pour procéder à des arrestations le plus souvent arbitraires ${ }^{7}$.

Le dialogue entre Djuhāa et les deux anges se poursuit, donnant lieu à une critique socio-politique bien acerbe. Il suffit, à cet égard, de considérer le « crime » dont les anges accusent le héros, à savoir : sauver sa peau aux dépens du bonheur des villageois en employant son intelligence pour son propre profit et non pour celui de la collectivité. Mais celui-ci se défend avec véhémence en ces termes :

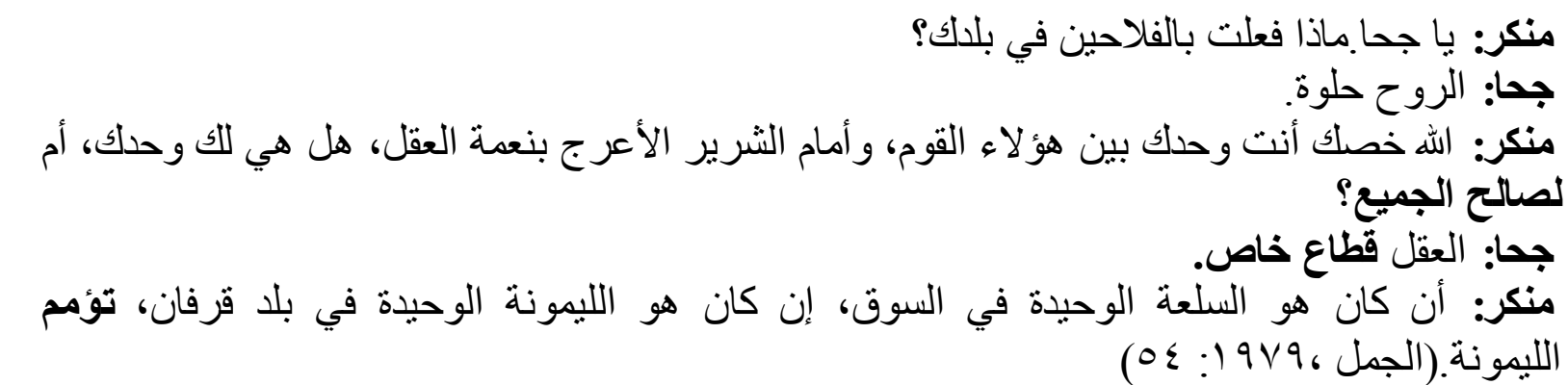

Mūnkar: Djuhā, qu'as-tu fais aux paysans, tes compatriotes?

Djuh̄ā : J'ai dû sauver ma peau.

Münkar : Parmi ces gens, et face au tyran boiteux, tu es le seul qu'Allah ait doté de la raison. Ce don est-il ton apanage exclusif, ou un bien mis au service de tous?

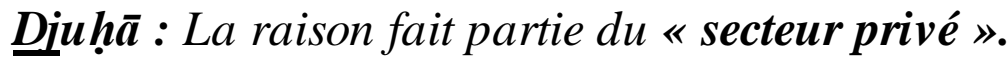

Münkar : Si c'est la seule marchandise disponible sur le marché et si c'est le bouquet, que ce bouquet soit nationalisé*. Puis, en note, l'auteur commente malicieusement l'usage du mot "nationalisé »

"تؤمم" :" من الكلمات التي هُرِّبت (بضم الهاء) في عكس اتجاه الزمن من عصر لاحق إلى عصر

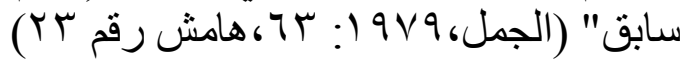

* "nationalisé » fait partie des mots qui se sont infiltrés à contre-courant d'une époque moderne à une époque antérieure. Ainsi dans l'exemple précédent, on note l'utilisation de termes qui renvoient à la vie socio-économique en Egypte moderne, notamment l'époque nasserienne caractérisée par une vague de 《nationalisation » et l'emprise du secteur public (11: 1999، جلال أمين). Déjà, Mūnkar, dans son discours, ne fait que reprendre un des clichés économiques qui sont à la base de toute politique économique socialiste : tout ce qui est utile à la société doit être mis au profit de la collectivité, même s'il s'agit des facultés intellectuelles de Djuhāa !

La note ajoutée par le narrateur ne fait que renforcer cette idée, en attirant l'attention du lecteur sur la contemporanéité du mot et, par conséquent, sur l'actualité de la critique. Si ces anachronismes sont facilement perçus par le LCont et Comp- et a fortiori par les LC et le LH-, il n'en est pas de même pour un L Non-Comp ou un lecteur moderne qui ignore l'idéologie économique des années soixante-dix. 
Outre l'allusion à la vie politique et économique contemporaine, le texte d'arrivée renferme aussi des références à certains aspects culturels de la société égyptienne ou arabe en général. A cet égard, une large place doit être réservée aux arrière-textes/intertextes religieux, à commencer par les versets coraniques explicitement ou implicitement cités dans le texte. Mais il s'agit souvent d'une « décontextualisation » de l'intertexte coranique, c'est-à-dire de son emploi dans un contexte bien différent du contexte originel. Ainsi, Djuhāa, portant une oie pour l'offrir à Tamerlan, se réjouit d'avance en pensant à la récompense escomptée du monarque ; d'où cette allusion, dans un contexte purement humoristique, à un verset coranique bien connu employé proverbialement. S'adressant à l'oie, il lui dit :

$$
\text { على الله تصطادى با سنارة با مطعمة، ياحسنة با اللى حتجيبى عشرة أمثالك. }
$$

Ah! Espérons que tu rapportes un gros gain, Ô toi, puissant aimant! Ô bonne action qui sera décuplée!

La dernière partie de cette phrase fait allusion au verset suivant :

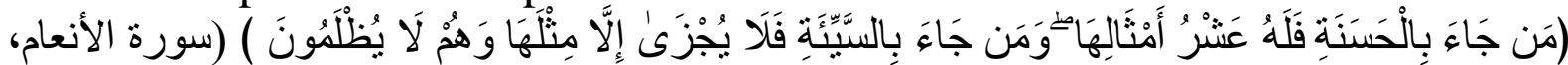

"Quiconque fait une bonne action recevra le décuplement (en récompense). Et quiconque commet une mauvaise action ne sera puni que de son pareil : ils ne subiront aucune injustice » (ABDELAZIZ, Zeinab, (2014), Sourate Al-An'Am) La référence à la culture islamique dépasse de loin une simple allusion à des versets coraniques. Nous avons déjà dit que l'action même du roman se passe pendant l'année dite "année de l'éléphant », surnom qui désigne principalement l'année de naissance du Prophète. Cette appellation est due à un événement historique mémorable : la tentative de démolition de la Ka'ba par l'armée d'Abraha l'abyssin, précédée d'un éléphant. Cette attaque s'est soldée par un échec, après l'extermination de l'armée abyssine grâce à des oiseaux lanceurs de pierres mortels envoyés par Allah. Cet épisode fait le sujet de la Sourate 105 : AL-FĪL (l'Éléphant)

La référence à "l'année de l'éléphant »fait déjà partie du titre même du roman arabe, mais elle ne sera explicitée que vers la fin du roman, lorsque les deux éléphants de Tamerlan, amenés au village selon le «conseil » de Djuhāa y font un grand ravage. Incapables d'arrêter ce désastre, les villageois mettent Djuhâa, auteur de cette catastrophe, devant ses responsabilités, et l'incitent à y trouver remède; celui-ci réussit habilement à tirer son épingle du jeu : assimilant le malheur des villageois à celui des habitants de la Mecque lorsque l'éléphant d'Abraha était sur le point de les attaquer, Djuhāa leur propose une solution « miraculeuse » basée sur un intertexte coranique bien évident :

$$
\begin{aligned}
& \text { أتت الوفود جحا. } \\
& \text { يا شيخ نصر الدين، ياصديقنا، و يا أيها العدو المبين (....) كما أوقعتنا انتشلنا. } \\
& \text { نحن يا أعز ائى في عام الفيل. } \\
& \text { (....) ما العمل؟ آعزب }
\end{aligned}
$$


- ليس أمامنا إلا صلاة (...) من نوع خاص. أسميها صلاة السجيل.

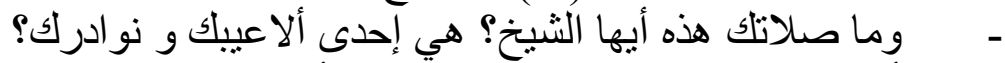

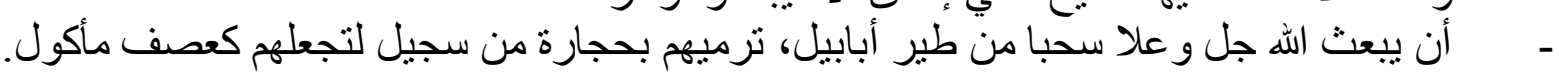

Des délégations arrivent chez Djuḥa

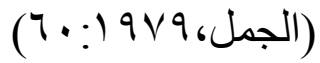

- $\quad \hat{O}$ Cheikh Nasrudīn, Ô notre ami et notre ennemi mortel. Retire-nous de cet embarras où tu nous as mis.

- Mes chers (villageois), nous sommes dans l'année de l'éléphant.

- Que faire donc?

- Tout ce que nous pouvons faire, c'est d'entamer une prière bien particulière, que j'appelle "la prière de marne ».

- $\quad$ Mais qu'est-ce que cette prière-là ? Est-ce un truc ou une anecdote de ta part?

- (Nous prierons) Allah le Très-Haut pour qu'Il envoie contre eux des oiseaux par volées leur lancer des pierres de marne entassées pour les rendre comme de la paille rongée.

Par cette allusion indirecte aux versets coraniques, Djuhāa annonce à ses concitoyens que, face au despotisme aveugle de Tamerlan, ils ne peuvent qu'implorer le secours de La Providence. Peut-être a-t-il jugé ses compatriotes trop faibles pour se soulever contre ce tyran : n'ont-ils pas toujours recours à Djuhā chaque fois qu'ils ont une demande à présenter à Tamerlan, le laissant seul faire face à ce despote ? L'allusion aux versets coraniques acquiert alors la valeur d'une critique amère indirectement adressée aux villageois. Cet intertexte est facilement décelable par un LS ayant la moindre connaissance de la culture arabo-islamique; mais le sens profond qu'il renferme ne sera probablement perçu que par les LC et LH.

Pourtant, d'autres intertextes culturels seraient plus difficilement repérés ou même compris, car ils sont liés à la littérature arabe ancienne, tel l'exemple de l'épigraphe même du livre. Or, Antoine Compagnon souligne que, par la " mise en épigraphe d'une citation », "le livre se donne pour la récriture d'une citation inaugurale et qui vaudrait pour le tout. » (Compagnon, 1979 :34) L'épigraphe joue donc le double rôle d'annoncer obliquement le sujet au lecteur et de résumer en quelque sorte toute l'œuvre. De même, en choisissant de citer un tel écrivain en épigraphe dans une œuvre, l'auteur dévoile une certaine tendance culturelle et, du même coup, choisit ses émules parmi les gens de lettres. "L'épigraphe, note Genette, est à elle seule un signal de culture, un mot de passe d'intellectualité, (...) elle est un peu, déjà, le sacre de l'écrivain, qui, par elle, choisit ses pairs, et donc sa place au Panthéon. " (Genette, 1987:148).En effet, Al-Djamal débute son roman par une épigraphe qu'il attribue à un des grands écrivains arabes classiques, à savoir : Ibn-al-Muķafa' :

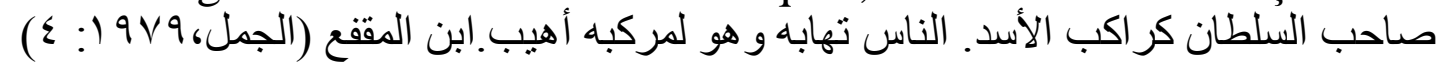


"Le compagnon du roi ressemble à celui qui monte sur un lion : tous les gens le redoutent, alors qu'il craint beaucoup plus sa monture. "Ibn-al-Mukafa' Cette citation inaugurale introduit et résume déjà la problématique de toute l'œuvre; à savoir : la relation tendue entre Djuhā et Tamerlan le monarque despote, relation pourtant enviée par les autres villageois. Quoique l'attribution de la citation arabe à Ibn-al-Muķafa' soit bien contestée ${ }^{8}$, le seul fait qu'AlDjamal cite cet auteur en tant que locuteur de cet énoncé marque bien qu'il cherche à établir une certaine similitude entre son œuvre et le livre le plus connu de ce fameux écrivain arabe, à savoir : Kalila et Dimna, le plus ancien recueil de fables arabes. Ainsi Al-Djamal classe-t-il son œuvre sur le même niveau qu'une œuvre bien connue, ce qui lui donne un certain prestige. Du même coup, il déclare indirectement que son livre appartient à la même veine de Kalila et Dimna; étant un récit symbolique au même titre que les fables d'Ibn-alMuķafa', la critique indirecte que renferme l'œuvre d'Al-Djamal peut passer impunément.

Placée juste après le titre sans explication aucune, l'épigraphe est un intertexte en attente d'une interprétation; elle n'acquerra toute sa signification que vers la fin du roman. Selon Genette, "épigrapher est toujours un geste muet dont l'interprétation reste à la charge du lecteur »(Genette, 1987: 145).Une question s'impose donc: comment les divers lecteurs comprendront-ils l'épigraphe d'Al-Djamal ? Alors que le LS se contentera de parcourir l'énoncé sans se donner la peine de réfléchir sur l'identité de son auteur, et sans chercher à établir un lien entre cet énoncé et le texte qu'il lit, le LC y verra une introduction à une histoire sur la relation roi/courtisan, et le LH, mieux informé, cherchera à comprendre le lien qui unit le texte d'Al-Djamal à l'œuvre d'Ibn-alMuķafa' d'où est extrait cet énoncé.

En choisissant ses intertextes ou arrière-textes, Al-Djamal reste accessible à un large public formé, pour la plupart, de LS, à part quelques arrièretextes/intertextes à caractère socio-politique, économique ou culturel. Du même coup, il ne prive pas les LC et LH de quelques intertextes /arrière-textes plus subtils à comprendre qui délectent leurs esprits. Ce dosage est propre à garantir au texte arabe un public assez large.

$\mathrm{Au}$ moment où le héros d'Al-Djamal représente une imitation assez fidèle mais modernisée de l'humoriste arabe le plus célèbre, un peu plus loin sur les rives de la Méditerranée, naît Moha, autre personnage inspiré de Djuhā qui, pourtant, prend ses distances avec son ancêtre folklorique. En effet, Moha est non seulement le fils légitime de la société marocaine qui l'a engendré, mais aussi le témoin certificateur de l'entrecroisement des deux cultures orientale et occidentale chez son créateur, Ben Jelloun.

II-Moha dans «Moha le fou, Moha le sage » :

Personnage plus complexe que le héros d'Al-Djamal, le Moha de Ben Jelloun est le bilan de l'enchevêtrement de plusieurs arrière-textes et intertextes. 
Du premier coup d'œil, on peut mesurer ce que doit le personnage de Ben Jelloun à son ancêtre folklorique Djuhāa : la dualité de caractère qui transparaît dès le titre même de l'œuvre, Moha étant un héros fou-sage. Tout le long de l'œuvre, il ne cesse d'émettre des jugements et d'accomplir des actes qui, sous un faux-semblant de folie, voilent une vision lucide du monde.

Pourtant, le héros de Ben Jelloun se distingue par sa mélancolie contrastant violemment avec tout l'héritage traditionnel de son prédécesseur folklorique et avec le personnage d'Al-Djamal aussi. Dès les premières pages du roman, Moha a bien déclaré : Je ne ris pas. Je suis triste et ravagé par l'inquiétude. »(Ben Jelloun,1978 :19) Aucune anecdote ne figure dans le texte marocain, et Moha ne s'adresse à ses compatriotes que sur un ton grave, voire solennel. De cette gravité découle un autre écart fort bien significatif entre le héros marocain et ses deux homologues folklorique et égyptien : au moment où Djuhāâ, l'humoriste arabe, ainsi que son successeur égyptien-le héros du roman d'Al-Djamal-, incarnent tous les deux l'homme du peuple un peu terre-à-terre qui cherche en premier lieu à sauver sa peau, Moha, plus idéaliste, se voue à une cause unique : combattre le Mal par sa parole. Ainsi, le héros de Ben Jelloun s'exclame : "Je passe ma vie à me battre avec des abstractions comme un Don Quichotte ; je suis dans une forêt sans arbres. » (Ben Jelloun, 1978 :123) Il se compare, à juste titre, au fameux personnage utopique de Cervantès : les deux rêvent leurs vies au lieu de la vivre. Cet arrière-texte purement occidental donne au personnage de Ben Jelloun une dimension plus large que celle de son homologue égyptien et le rend accessible à un public plus étendu : un LNonComp assez cultivé.

Mais l'arrière-texte/intertexte occidental lié au personnage de Moha est parfois très subtil à déceler. Dans une sorte d'extase, le héros de Ben Jelloun s'écrie : «moi, je dis tout ; je suis assailli de messages et d'informations. (....) Je vadrouille. (...) Le corps plein de cris étouffés... Ainsi parlait Moha. » (Ben Jelloun, 1978 : 49,50).Or, dans son étude consacrée à "l'esthétique de la folie » dans les littératures maghrébine et négro-africaine, Kahina Bouanane note que la dernière phrase rappelle le titre du fameux ouvrage "Ainsi parlait Zarathoustra», soulignant ainsi la similitude entre les deux héros, celui du philosophe allemand et celui de l'écrivain marocain "Tout comme Nietzsche ,écrit Bouanane, Ben Jelloun met à l'œuvre un sage solitaire qui tente d'éveiller les hommes de leur profond sommeil. » (Bouanane, 2012 :60) Or l'arrière-texte de Nietzsche étant un ouvrage philosophique connu par les spécialistes, il est fort probablement ignoré du LS, assez connu par certains LC ; c'est le LH qui, grâce à ses recherches méticuleuses, pourra saisir l'allusion à l'œuvre nietzschéenne.

Notons aussi que le personnage de Moha puise ses racines dans un autre arrière-texte non-fictionnel, ancrée dans l'histoire marocaine. En effet, « Moha » est le surnom d'un héros marocain de chair et d'os appelé Hamou Zayani, une 
figure de proue de la résistance à l'occupation française. "C'est un des plus grands hommes berbères que l'imaginaire marocain, surtout en moyen Atlas, mythifie. (...) Les Français n'établissent réellement leur autorité sur les tribus zayanes, qu'à la mort de Moha ou Hamou en 1921, les armes à la main, tué lors d'une bataille contre les troupes françaises dans le Moyen Atlas.» (Fdil, 2014 :6-7) Ce qui confirme en plus le lien entre Moha (Hamou) et le héros éponyme de Ben Jelloun, c'est que celui-ci se vante du rôle qu'il a joué dans la résistance à l'occupation française, en transmettant des messages aux résistants et en passant les frontières avec des armes. (Ben Jelloun, 1978 :113-114) Cette image de Moha en guerre directe contre les colonisateurs contraste fortement avec la tradition folklorique de Djuhāa, qui, lui, n'a jamais porté des armes dans les textes qui lui ont été attribués. Pourtant, un tel intertexte fort ancré dans la tradition marocaine ne sera perçu que par un LComp ou par un LH qui cherche à approfondir ses connaissances sur l'histoire du Maroc.

En somme, Ben Jelloun a, semble-t-il, créé son héros grâce à une gamme d'arrière-textes les plus variées, puisées au folklore arabe, à l'histoire marocaine, avec des réminiscences de la philosophie nietzschéenne ou d'autres influences occidentales. Dans son étude intitulée : "Le discours de la folie dans Moha le fou Moha le sage ", Ait Allaoua note que ce choix n'est pas sans influence sur le lectorat du roman "Cette attitude, note-t-elle, montre une volonté d'impliquer les deux lecteurs, l'occidental et le maghrébin, afin de les rapprocher et de créer un dialogue entre les deux rives à travers ces personnages mythiques partagés» (Ait Allaoua,, 2009 :52) Or, cette conclusion exige a fortiori que les deux lecteurs, occidental et oriental, soient capables de déchiffrer les arrière-textes/intertextes inhérents à la création de ce personnage pour en déceler les éléments qui appartiennent aux deux cultures orientale et occidentale, ce qui n'est pas souvent le cas, à moins qu'il ne s'agisse d'un LH qui s'aventurerait à suivre, à perte de souffle, les traces intertextuelles dans la création de ce personnage.

Mais d'autres éléments, appartenant au cadre spatio-temporel du roman, font de Moha un citoyen marocain par excellence. Ben Jelloun, sans préciser ouvertement que l'action se passe au Maroc, ne manque pas de parsemer son œuvre de quelques arrière-textes/intertextes qui font allusion à des réalités marocaines ou, plus généralement, maghrébines. Il est à noter que Ben Jelloun s'efforce, tout au long de l'œuvre, de laisser son lecteur indécis quant à la diégèse du roman, en lui offrant trois types différents d'arrière-textes/intertextes qui tracent le cadre spatial de l'œuvre: le premier est teint d'une couleur fortement marocaine, le deuxième évoque un espace tunisien ou algérien, et le troisième situe l'action dans l'un des trois pays du Maghreb sans aucune précision. Dans un énoncé, Moha adresse la parole à ses concitoyens, faisant allusion à la réalité purement algérienne, en ces termes: «Le million de martyrs ne te suffit pas?» (Ben Jelloun, 1978 :131) Quelques pages plus loin, il déclare : 
«je hurlais contre la présence des Français chez nous (...) c'était dans la casbah. » (Ben Jelloun, $1978: 135$ ), ce qui renvoie clairement à une ville marocaine. Plus tard, Moha, dont les paroles inquiétaient les autorités, est arrêté par la police et soumis à l' examen d'un psychiatre pour évaluer ses compétences intellectuelles et déceler les marques de folie chez ce héros. Le médecin lui "pose une question simple : où sommes-nous ici ? ", Moha répond : "Nous sommes à Tlemcen. Oui, c'est ça, je reconnais, nous sommes à Salé. Tlemcen. Oui, c'est bien ce que je pensais. Sfax. Non, je me trompe. Nous sommes peutêtre dans un cimetière sans nom, sans pays. » (Ben Jelloun, 1978 :151) Cet enchevêtrement de noms de villes qui appartiennent aux trois pays du Maghreb marquera, pour un LC ou un LH, moins un indice de la folie de Moha qu'un subterfuge pour adresser une critique indirecte à la fois aux trois pays en question, d'autant plus que Moha termine son discours en comparant le lieu où il se trouve à « un cimetière sans nom, sans pays. ».

$\mathrm{Si}$ les arrière-textes/intertextes spatiaux et historiques sont puisés conjointement des trois pays du Maghreb, ceux qui relèvent du domaine culturel sont typiquement marocains, tels que les proverbes. Comme le Djuhāa d'AlDjamal, le Moha de Ben Jelloun cite des proverbes sans les désigner en tant que tels dans la plupart des cas, ils ne seront donc compris que par un L Comp ou un LH. Par exemple, tout à fait au début du roman, quand Moha s'adresse à un interlocuteur non défini pour lui expliquer les grandes lignes de sa «philosophie », il lui dit: «Je surveille la lune et je survole le territoire. C'est la lune qui fait les vagues.(...) Tu connais, toi, le secret des vagues? » A ce stade, jugeant son interlocuteur incapable de saisir le sens de ses paroles, Moha poursuit : "Oui, c'est vrai! Que comprend l'âne au gingembre? Et l'homme à l'écume?» (Ben Jelloun, 1978 :23) Cet énoncé reprend littéralement un

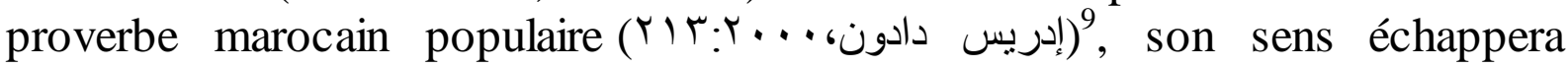
probablement à tout LNon-Comp.

A part les proverbes, il existe chez Ben Jelloun un autre type d'intertexte culturel difficile à déceler, à savoir : l'intertexte religieux qui peut passer presque inaperçu pour un lecteur n'ayant pas une bonne connaissance de la culture musulmane. Ainsi, Moha, voulant donner à ses concitoyens un exemple à suivre, s'écrie : "Pourquoi vous ne mangez pas du foin comme nos ancêtres issaoua? Eux n'avaient jamais peur. Ils savaient se fendre le crâne avec une hache et boire le sang de leur cervelle. Ils savaient rire et tourner en dérision la mort. » (Ben Jelloun, 1978 : 31) Cet énoncé fait référence à "Al-Aïssawiyya », confrérie marocaine relevant du soufisme et fondée au $X V^{\mathrm{e}}$ siècle sous l'impulsion du cheikh Muhammad Ben Issa ${ }^{10}$. Or, cet arrière-texte purement marocain ne sera compris que par un LComp ou un LH qui s'évertuerait pour se renseigner sur cette confrérie, surtout que le texte ne fournit aucune indication de nature à guider ses recherches. 
D'autre part, Moha, tout au long de l'œuvre, ne cesse d'évoquer un personnage assez connu de la tradition islamique : Al-Mahdi (ou Mahdi tout court). Il le cite la première fois pour mettre ses concitoyens en garde du sort tragique qui les attend s'ils se résignent à accepter l'injustice; il le présente vaguement à ses interlocuteurs : "J'ai peur de vous voir pendus à l'aube de tous les massacres. (...). J'entends la voix de Mahdi. (...) Il va surgir comme le prophète, un prophète armé et vengeur. Mahdi est une légende pour vous faire attendre. Attendre toute une vie. Attendre la mort, assis au soleil, les jambes croisées, le dos contre le mur, la tête entre les mains» (Ben Jelloun, 1978 :32,33) Notons que, selon la tradition prophétique, Mahdi viendra à la Fin du monde, après une assez longue période d'injustice, pour répandre l'équité sur la terre ${ }^{11}$. Or Moha semble plutôt critiquer la croyance au retour du Mahdi, en la qualifiant de "légende »destinée à "faire attendre » les gens, à les inciter à l'inaction jusqu'à "la mort». Moha va plus loin encore : dans un élan d'extase débridée, il déclare : "Mahdi, c'est moi...Je m'appelle Mahdi, l'homme investi de la mission suprême (...) J'arrive après toutes les catastrophes. Je vous annonce le malheur. » (Ben Jelloun, 1978 : 67,68) Ainsi, Moha déclare qu'il est devenu un Mahdi « à rebours », qui vient annoncer aux gens, non pas une ère d'équité, mais plutôt «le malheur». Un lecteur mal informé pourrait bien se tromper sur la véritable signification de l'arrivée de Mahdi sur terre telle que la stipule la tradition islamique. Dans un autre énoncé, Moha s'écrie : «Mahdi! Quand tu reviendras, personne ne te reconnaitra...meurs de nouveau...vat'en ...laisse cette foule à sa paresse. »(Ben Jelloun, 1978, p.69). Un LC ou un LH bien avertis comprendront qu'il s'agit non pas d'un refus de l'arrivée de ce personnage, mais plutôt d'un cri de désespoir, car, selon Moha, l'humanité souffre trop d'une "paresse » séculaire pour qu'elle mérite la justice qui règnera avec l'arrivée de Mahdi.

Dans l'étude de l'intertexte religieux chez Ben Jelloun, une place à part doit être réservée à l'analyse des versets coraniques. En effet, les intertextes coraniques parsèment l'œuvre, mais, la plupart des cas, sans les désigner en tant que tels, comme dans l'exemple suivant. Evoquant la relation intime entre les femmes et les hommes, Moha déplore en ces termes le sort des femmes assujetties, selon lui, à se plier aux caprices des hommes : " $\hat{\text { ofemmes, }}$ pourquoi vous cultivent-ils dans les ténèbres avec des sexes en bois, sans caresses, sans tendresse? (...) je pense à vous. Insatisfaites, cultivées, labourées par des siècles de silence et de brutalité légalisée par l'Autorité suprême. (...) «La femme est un champ à cultiver...»C'est vrai. C'est un champ. Mais un champ vivant, en droit d'exiger autre chose que la fêlure systématique et semence brève. » (Ben Jelloun, 1978 : 46 à 48) Dans ce passage, il faut d'abord souligner la présence d'un énoncé cité entre guillemets : "La femme est un champ à cultiver...» Déjà, utiliser ce signe typographique pour encadrer un énoncé signifie, selon Antoine Compagnon, que «la parole est donnée à un autre, que 
l'auteur se démet de l'énonciation au profit d'un autre : les guillemets désignent une re-énonciation ou une renonciation à un droit d'auteur. "(Compagnon, 1979 :40) Pourtant, dans l'énoncé précité, aucune source n'est mentionnée pour indiquer au lecteur d'où cet énoncé est puisé. Or, si l'on considère l'emploi répétitif du verbe «cultiver » et de son synonyme «labourer » pour décrire la relation intime entre homme et femme, ainsi que l'allusion à une «Autorité suprême » qui a légalisé cette relation, on peut conclure que l'énoncé « $\boldsymbol{L} \boldsymbol{a}$ femme est un champ à cultiver..." fait bel et bien allusion au verset coranique: «Vos femmes sont un labour pour vous. Allez-donc à votre labourage comme vous voulez, et faites-vous précéder de bonnes actions. Craignez Allah, sachez que vous le rencontrerez. Et annonce une bonne nouvelle aux croyants." (ABDELAZIZ, Zeinab, 2014, Sourate La Vache, verset 223) ${ }^{12}$ Pourtant, Moha donne à ce verset une interprétation bien étriquée mais assez répandue en Occident ${ }^{13}$, qui est bien loin de celle donnée par les différents exégètes du coran ${ }^{14}$. En tous les cas, cet intertexte coranique, sournoisement inséré dans le texte de Ben Jelloun, serait loin d'être perçu par un LS ou un LC ; seul un LH, après des recherches, saura noter la présence d'un tel intertexte et apprécier la hardiesse de la critique contenue dans cet énoncé.

Si l'intertexte religieux chez Ben Jelloun est bien difficile à saisir et à analyser, plus ardu encore est l'arrière-texte/intertexte culturel qu'il insère dans son texte. Cet auteur bilingue, imbu d'une double culture orientale et occidentale, ne cesse de parsemer son œuvre d'arrières-textes/ intertextes puisés à ces deux cultures. Ainsi, un riche capitaliste expose à Moha sa doctrine pragmatique basée sur un calcul froid des bénéfices; puis il critique en ces termes Moha qui cherche à changer la vie des autres à l'aide des mots : "Le pays n'a pas besoin de mots, surtout pas de poésie, il a besoin de progrès et de techniques nouvelles. La poésie c'était bon du temps des Abbassides, du temps de l'Andalousie, du temps de l'accalmie et des lauriers de l'histoire. » (Ben Jelloun, 1978 :97) Dans cet énoncé, la référence à deux époques célèbres de l'histoire islamique présuppose que le lecteur a déjà une idée assez bonne de ces périodes pourtant assez longues ; il est donc destiné à un LC ou un LH, notamment d'origine arabe.

Alors que la référence culturelle dans l'exemple précédent est assez explicite, un autre cas est plus ambigu, d'autant plus qu'il s'agit d'un intertexte mis en épigraphe au début du roman. Le voici :

"O muets, muets cimetières en vos tristes allées,

je hurle, je crie ;je crie, me lamente (...)

(Badr Châker as-Sayyâb, (traduit par André Miquel) » (Ben Jelloun, 1978 :7)

En effet, Ben Jelloun choisit de citer un poème écrit par un poète irakien, Badr Châker as-Sayyâb (1926-1964), connu par ses positions d'avant-garde ${ }^{15}$. Or, ces vers imprégnés de tristesse, mis en tête de l'œuvre de Ben Jelloun, donne à celle-ci une tonalité douloureuse qui contraste avec l'héritage folklorique de 
Djuhā, mais qui annonce déjà le caractère de Moha. Or, le fait même de citer un tel poète, connu surtout par l'élite intellectuelle du monde arabe et fort peu connu des LS, surtout les lecteurs francophones, Ben Jelloun n'a-t-il pas, dès les premières lignes de son œuvre, choisi son lectorat ? Il a, du moins, mis en garde son lecteur, en lui annonçant que son texte sera aussi profond et obscur que ces vers. Cette épigraphe est la meilleure illustration de cet énoncé d'Umberto Eco : "Les épigraphes semblent introduire quelques complications : elles sont hermétiques. Mais à la première lecture on les laisse tomber (n'agit-on pas ainsi d'habitude?)»(Eco, 1985: 261) Et si le LS se suffit déjà de cette " première » et unique lecture, soit il va «laisser tomber » cette épigraphe, trop ardue pour qu'il la comprenne, soit il renoncera d'emblée à poursuivre la lecture de ce roman qui s'avère pour lui, dès les premières lignes, d'un abord assez difficile. Par contre, un LC et un LH trouveront dans cette épigraphe, où se côtoient l'implicite et l'incertain, un stimulant pour poursuivre une lecture érudite du roman.

Un peu plus loin dans son roman, Ben Jelloun cite une autre épigraphe attribuée à un poète français qui offre beaucoup de ressemblance avec Badr Chaker Es-Sayyab: Georges Bataille. C'est l'auteur d'un bon nombre de poèmes et de nouvelles analysés par les critiques mais qui, comme le note Isao OKI, «attirent rarement l'attention des lecteurs. » (Oki, n.d. :73) Ainsi, Ben Jelloun, après avoir décrit l'enterrement de Moha, met en épigraphe, avant le chapitre suivant, ces vers de Bataille :

\section{«(...) je parle de chez les morts}

et les morts sont muets. » (Ben Jelloun, 1978 :159)

Juste après ces vers, Moha, enterré, commence à parler à ses concitoyens ; le vers de Bataille : «je parle de chez les morts » est donc pris au pied de la lettre, l'épigraphe formant ainsi un prélude à la dernière partie du roman. Or, ce sens sera plutôt saisi par un LC ou un LH susceptibles de comprendre l'épigraphe à la lumière du chapitre suivant. Quant au LS, il pourrait bien ignorer aussi bien le sens de l'épigraphe que la biographie de son auteur.

Ben Jelloun va plus loin encore, en empruntant au grand philosophe allemand Nietzsche une citation qu'il met à la fin du roman.

"Je ne suis qu'un faiseur de mots

Quelle importance ont donc les mots?

Et moi, quelle importance ai-je donc? » (Ben Jelloun, 1978 :186)

Comme Al-Djamal, Ben Jelloun termine son roman par un intertexte bien déroutant, qui peut donner lieu à plusieurs interprétations. Un LS s'arrêterait-il sur la signification de ces vers ou bien les laisserait-il tomber, tout comme l'épigraphe ? Comment un LC et un LH interpréteront l'interrogation dans ces vers ? Y verront-ils un signe de désespoir ? ou plutôt une déclaration d'échec de la part de Moha qui reconnaît l'inanité de tous ses efforts visant à combattre le mal par ses paroles? 
Notons aussi que l'auteur marocain insère dans son œuvre un autre intertexte attribué, cette fois, à un personnage fort peu connu : un chef indien des Etats Unis ! Ainsi, Moha, vivant en parfaite communion avec la nature, rapporte à son interlocuteur non défini des paroles qu'un certain Red Jacket a adressé à l'arbre qu'il vénère. (Ben Jelloun, 1978 :174) Non content de citer des écrivains arabes et européens, Ben Jelloun cite donc un énoncé qu'il attribue à ce personnage, sans même se soucier de le présenter à son interlocuteur. En effet, Red Jacket est le surnom d'un personnage réel appelé Sagoyewatha (1758?1830), chef d'une tribu de Peaux-Rouges et brillant orateur qui a joué un rôle important pendant la guerre d'indépendance des Etats-Unis ${ }^{16}$. Or, cet orateur indien est fort peu connu par un LS, qu'il soit oriental ou occidental. Un LH seul pourra, après avoir effectué ses recherches, connaître l'identité de ce chef indien et la place importante assignée à l'arbre dans la croyance des Peaux-Rouges. Ayant démontré une bonne érudition en ce qui concerne les cultures arabe et occidentale, Ben Jelloun fait preuve d'un savoir plus étendu, qui englobe aussi la culture américaine. Aussi risque-t-il de n'être totalement compris que de ses émules.

En somme, le Moha de Ben Jelloun se distingue nettement de son modèle folklorique ; il n'en garde que des traces à peine perceptibles. Il est le bilan de l'entrecroisement de la tradition orientale teinte d'une couleur marocaine et de la culture européenne qui confère à ce héros une portée universelle. 


\section{Conclusion}

Cette étude s'est proposée de mettre en application une théorie assez récente et fort intéressante pour toute recherche portant sur l'intertextualité ; cette nouvelle conception est basée sur la distinction entre l'intertexte et l'arrière-texte. En effet, la notion de l'arrière-texte, en englobant tout ce qui est en amont d'un texte, comble certaines lacunes dans les anciennes théories intertextuelles. L'apport de cette notion se révèle d'autant plus important qu'il s'agit d'une étude basée sur les réminiscences de la tradition orale dans certains textes modernes. L'étude de tels éléments dépasse le cadre restreint de la notion d'intertexte.

D'autre part, cette étude vise à mettre en évidence le lien étroit entre cette théorie et celle du Lecteur Modèle élaborée par Umberto Eco. En effet, les théoriciens qui ont étudié l'arrière-texte/intertexte et son insertion dans un texte n'ont pas accordé une grande importance à la détermination du type de lecteur apte à découvrir ces traces intertextuelles. De son côté, Eco a eu le mérite de jeter une lumière sur le rôle du destinataire dans l'interprétation et la genèse même du texte, sans pour autant mettre l'accent sur le rôle spécifique que joue l'intertexte/arrière-texte dans la détermination du type du lecteur potentiel de chaque texte. Il est donc bien évident que ces deux théories peuvent se compléter pour déterminer le mode de réception d'une œuvre. De même, cette étude a tenté d'élucider l'existence de différents types de lecteurs, autres que ceux mentionnés par Eco. Ainsi l'éloignement dans l'espace et /ou le temps joue-t-il un rôle important dans l'assimilation du texte par le lecteur : un LComp comprend des arrière-textes/intertextes relatifs à sa société mieux qu'un LNonComp ; de même, un LCont saisit l'allusion à certaines réalités politiques et économiques de son époque plus qu'un LNon-Cont. Un intertexte /arrière-texte peut être assimilé à une pierre de touche permettant d'identifier le(s) type(s) de lecteur(s) à qui s'adresse le texte en premier lieu.

En appliquant les théories de l'arrière-texte/intertexte et du Lecteur Modèle sur les deux corpus étudiés, nous pourrons conclure que le texte d'AlDjamal répond aux goûts des différents lecteurs potentiels de l'œuvre : il est accessible à la plupart des LComp et LCont, parfois moins accessible à un LS arabe moderne. De leur côté, les LC et les LH trouveront dans certains arrièretextes/intertextes une matière féconde qui puisse satisfaire leur goût pour une lecture critique, voire érudite. En ce qui concerne l'œuvre de Ben Jelloun, l'analyse de l'arrière-texte/intertexte montre que cet écrivain s'adresse moins au large public francophone qu'à des LComp. Pour plus de précision, disons que cet auteur francophone, jouissant d'une double culture orientale et occidentale, s'adresse, à plusieurs reprises, à son homologue, c'est-à-dire un lecteur érudit, de culture franco-arabe, aussi vise-t-il, en premier lieu, les LH et les LC. Quant au LS francophone moderne qui lit l'œuvre de Ben Jelloun, il aura le choix entre deux attitudes bien différentes :il peut laisser tomber certains énoncés 
intertextuels de son texte et continuer à lire le roman malgré une certaine incompréhension, ou bien renoncer d'emblée à poursuivre la lecture du roman.

Le débat est loin de se borner à la question -bien cruciale- de savoir à quel lecteur tel écrivain écrit. Grâce au fusionnement des deux théories précitées, on peut systématiquement trouver dans chaque texte des indices probables de sa viabilité auprès des générations futures. Cette constatation serait basée sur un postulat bien simple: plus une œuvre est parsemée de traces d'intertextes /arrière-textes fortement liées à un cadre spatio-temporel déterminé, moins elle sera comprise par un LNon-Comp et/ou un LNon-Cont, à moins qu'elle ne soit accompagnée d'un glossaire ou d'une quelconque annotation explicative, ce qui n'est pas toujours le cas.

Pour terminer, disons que tout lecteur moderne des deux romans en question, quelque soit son origine ou son niveau culturel, ne manquera pas de trouver un certain plaisir à relire Djuhāā, l'Humoriste arabe le plus célèbre. Ce personnage folklorique, devenu l'emblème des périodes de transition, incarne l'homme du peuple qui se venge de ses oppresseurs avec la seule arme de la satire mordante. Djuhāā est et sera toujours un héros universel, une moule apte à être façonnée par tout écrivain à toute époque. A chaque nouvelle reproduction, on lui donne, grâce à quelques traces intertextuelles, la saveur d'une région bien précise, d'une époque bien déterminée; ces traces ne font que broder la «texture » folklorique de Djuhāa en l'éclairant sous un jour nouveau. C'est là que réside le vrai génie de cet humoriste intarissablement ressuscitable. 


\section{$\underline{\text { Notes }}$}

${ }^{1}$ Nous adoptons dans cette recherche le système de translitération de l'« Encyclopédie de l'Islam ».

${ }^{2}$ Pour les citations puisées de ce livre, et de tout autre ouvrage arabe, nous les avons traduites tout en insérant le texte original arabe soit dans le corpus de notre étude (pour l'œuvre d'Al-Djamal), soit dans les notes (pour les ouvrages de référence.)

3 Voici le texte original :

"فكاهات عهود التحول": (....)وتأتى هذه الفكاهة في أو انها حين تؤذن العهود بالتحول لتز عزع أركانها

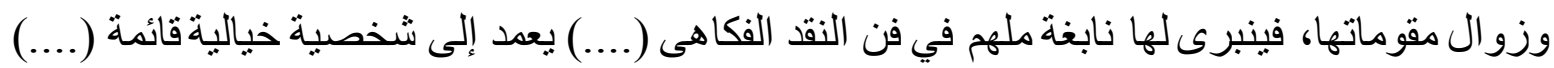

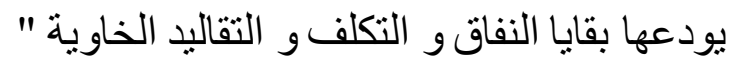

${ }^{4}$ Voici le texte original, suivi de l'interprétation d'Ahmad Taymūr Pacha :

"قالو ا يا جحا كلبك بالسخونه، قال أهو فاضى لها"

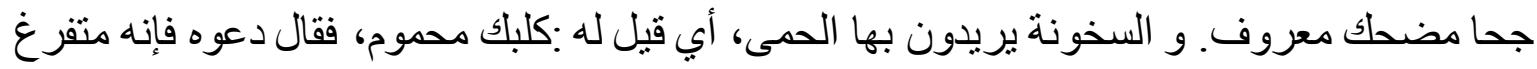

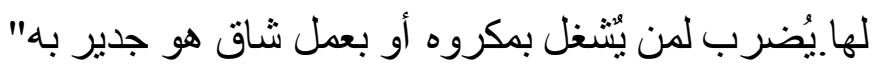

${ }^{5}$ Voici le texte original

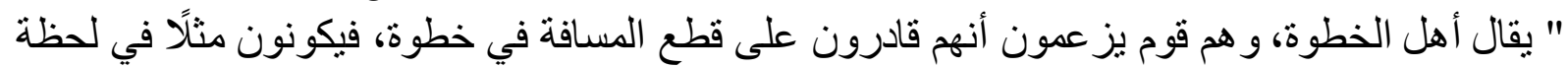

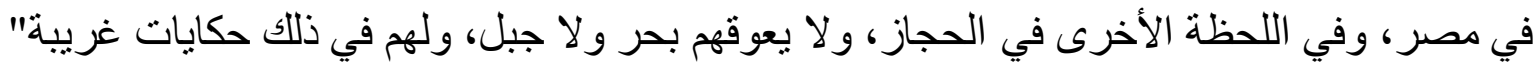

${ }^{6}$ Voici un texte explicitant l'évolution du sens de ce mot :

"طز" (...)هي خير تعبير عن اللامبالاة، تقال حينما يستهين الثخص بموقف ما، يستخدها المصريون

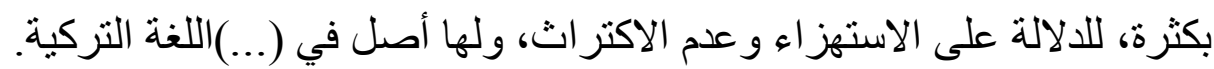

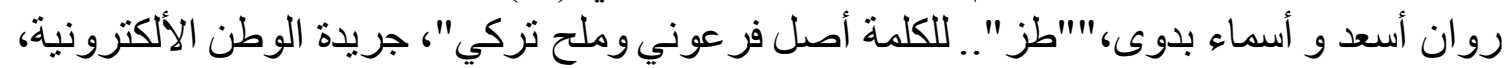

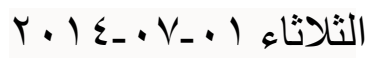

https://www.elwatannews.com/news/details/512925

${ }^{7}$ Pour plus de détails sur ces troupes, cf, entre autres :

"تعبير لازوار الفجر 《، الذي شاع استخدامه علي نطاق واسع في الصحافة المصرية حتى الآن، (...)

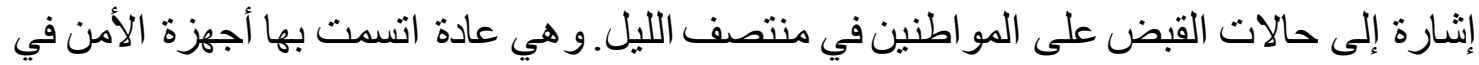

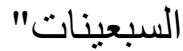

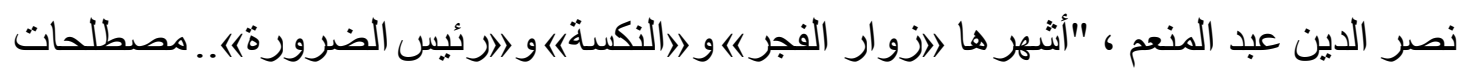

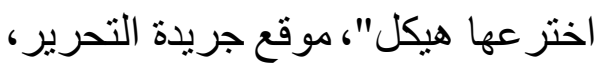

https://www.tahrirnews.com/posts/384248/

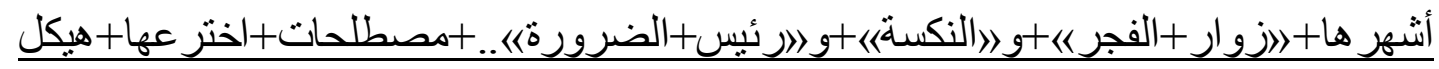


${ }^{8}$ Dans les ouvrages de référence de la littérature arabe classique, on cite cette citation comme étant un exemple de la sagesse universelle, sans pour autant l'attribuer à un auteur particulier. Cf., entre autres,

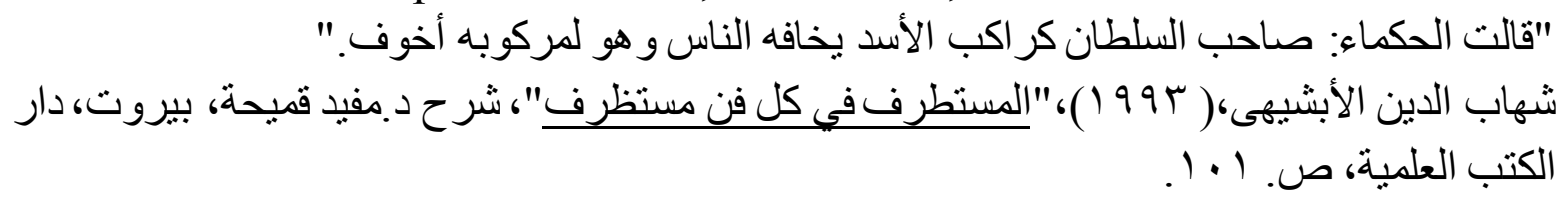

${ }^{9}$ Voici le proverbe en dialecte marocain :

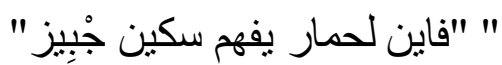
سكين جُبِيز: نوع من تو ابل الأطعمة يلذذها. يُضرب المثل لمن لا يعرف قيمة بعض الأشياء و مز اياها"

${ }^{10}$ Pour plus de détails, Cf le site de la confrérie :

http://confrerieaissawa.free.fr/html/francais/historique.html

${ }^{11}$ Pour plus de détails, cf :

Ibn Al-Qayyim et Al-Bustawy, (2008), Le Mahdîtantattendu! Traduction et adaptation : Karim Zentici, publié par Le bureau de prêche de Rabwah (Ryadh). Repéré à : https://d1.islamhouse.com/data/fr/ih_articles/fr-

LeMahditantattenduZentici.pdf

${ }^{12} \mathrm{Cf}$ le verset originel :

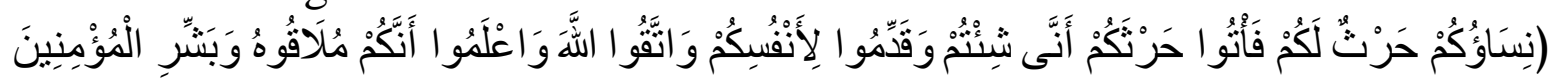

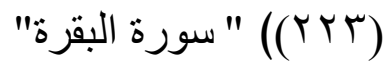

${ }^{13} \mathrm{Cf}$, à titre d'exemple, l'interprétation suivante :

«La métaphore du labour est le plus souvent comprise sexuellement: l'homme pourrait aller comme il veut à sa terre que serait sa femme pour la féconder. Celle-ci semblerait ne pas avoir voix au chapitre. Elle appartiendrait à son époux comme une propriété privée dont il pourrait disposer comme il voudrait. Le désir de l'épouse ne serait pas pris en compte. Elle devrait se résigner à être un objet sexuel passif de l'homme.

Pierre TRIGANO, "Le Coran et les femmes », article en ligne http://www.pierre-trigano.com/2018/01/06/le-coran-et-les-femmes/

${ }^{14}$ Pour plus de détails sur l'interprétation complète du verset par les exégètes du coran, cf : 
https://library.islamweb.net/newlibrary/display_book.php?flag=1\&bk_no=49\&I $\underline{\mathrm{D}=190}$ et https://vb.tafsir.net/tafsir6029/\#.XRVpYegzbIU

${ }^{15}$ Pour plus de détails sur ce poète, $\mathrm{cf}$ :

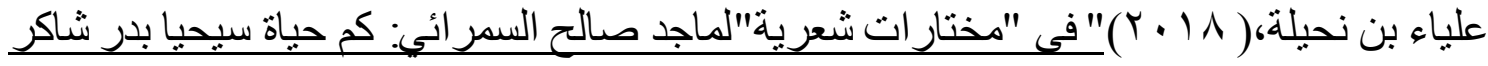

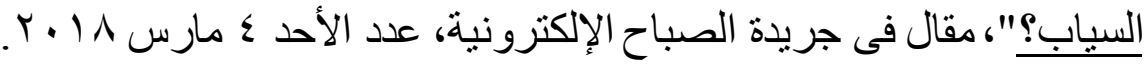

http://www.assabah.com.tn/article/149891/

${ }^{16}$ Pour plus de détails, cfl'article «Red Jacket » dans « Encyclopaedia Britannica »

https://www.britannica.com/biography/Red-Jacket-Seneca-chief 


\section{Bibliographie}

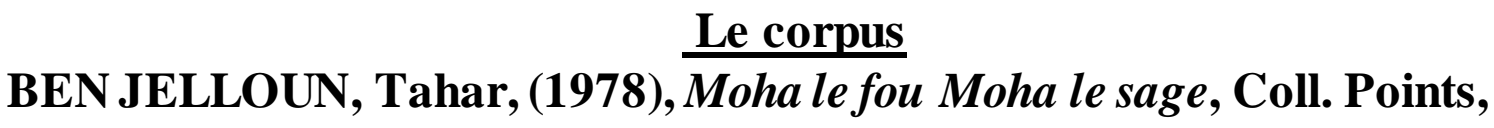
Paris, Ed. Seuil.

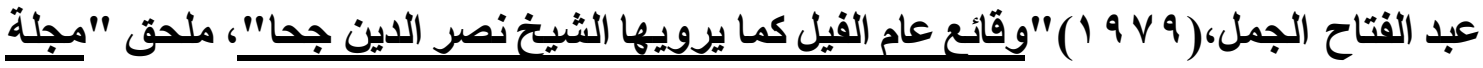

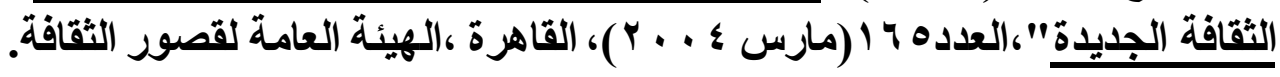
Bibliographie

ABDELAZIZ, Zeinab, (2014), Le Qur'ān, Traduction du sens de ses Versets , repéré à

http://www.zeinababdelaziz.com/pdf/Quaran/Traduction\%20du\%20Qur\%E2\% 80\%99\%C4\%81n\%20Prof\%20Dr\%20Zeinab\%20Abdelaziz\%202014.pdf

AIT ALLAOUA Kahina, (2009), "Le discours de la folie dans Moha le fou Moha le sage de Tahar Ben Jelloun », Mémoire de Magistère, Faculté des Lettres et des Sciences Humaines, Département de Français, Université Mouloud MAMMERI.

BONN, Charles, (1986), Problématiques spatiales du roman algérien, Alger, E.N.A.L. 115 p.

BOUANANE, Kahina, (2012), L'esthétique de la folie au sens « sur-réaliste » et sa dynamique dans l'espace de l'écriture, Topique 2012/2 (n 119), 53-69.

BOUGUEBINA, Fouzia, (2010) «Lecture et réception du texte maghrébin de langue française », Revue Académiques des Etudes Sociales et Humaines, 32010, 3-8.

CHAMS, Zinat, (2002), La Fontaine et ses sources orientales, Thèse de doctorat, Faculté des Lettres, Département de Langue et de Littérature Françaises, Université de Helwan.

CHANFRAULT, Bernard, (1991) Le corpus de Gafsa, Le cheval de Troie, $\left(\mathrm{n}^{\circ} 4\right.$, septembre), "Littératures orales : Arabes, Juifs et Siciliens », 13-19.

COMPAGNON, Antoine, (1979), La seconde main ou le travail de la citation, Paris, Editions du Seuil.

ECO, Umberto (1985), "Lector in fabula. Le rôle du lecteur ou la coopération interprétative dans les textes narratifs. », traduit par Myriem Bouzaher, coll. Biblio essais, Le Livre de Poche, Editions Grasset \& Fasquelle.

ECO, Umberto (1992), «Les Limites de l'interprétation », traduit de l'italien par Myriem Bouzaher, Editions Grasset \& Fasquelle. 
FDIL, Abdellatif, (2014), L'engagement des Marocains au cours du 1er conflit mondial entre histoire et représentations imaginaires, $11^{\text {ème }}$ colloque International Interdisciplinaire de Sorèze, Feb., Sorèze, France.p.6,7. Repéré à : <http://lara.hypotheses.org/709>. 〈hal-01265072>

GENETTE, Gérard, (1982), Palimpsestes. La littérature au second degré, Paris, Editions du Seuil.

GENETTE, Gérard, (1987), Seuils, Paris, Editions du Seuil.

MEMMI, Albert, (1991), L'ingénieux, Le cheval de Troie, ( ${ }^{\circ} 4$, septembre), «Littératures orales : Arabes, Juifs et Siciliens », 55-62

OKI, Isao,(n.d .), La Poétique de Georges Bataille et la notion d'informe, 7389, repéré à :https://www.jstage.jst.go.jp/article/ellf/108/0/108_73/_pdf/-char/ja RABAU, Sophie, (2002), L'intertextualité : introduction, choix de textes, commentaires, vade-mecum et bibliographie, SL, éd Flammarion.

TROUVE, Alain (2018), "Nouvelles déclinaisons de l'arrière-texte », Reims, Editions et Presses Universitaires de Reims (EPURE)

\section{المراجع العربية}

إبراهيم أحمد شعلان (ب9 99 ()،"النو ادر الثتبية المصرية، در اسة تاريخية إجنماعبة"، القاهرة، مكتبة

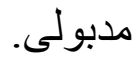

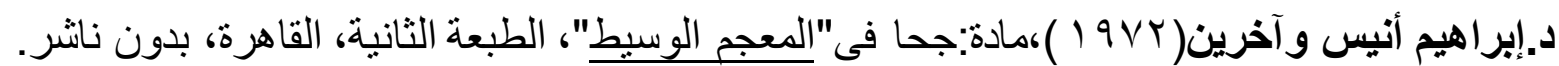

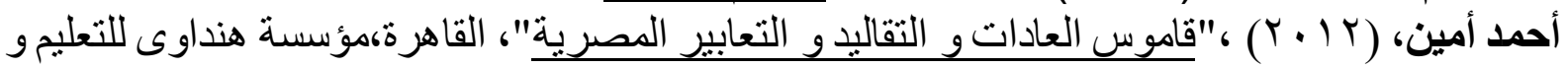
الثقافة.

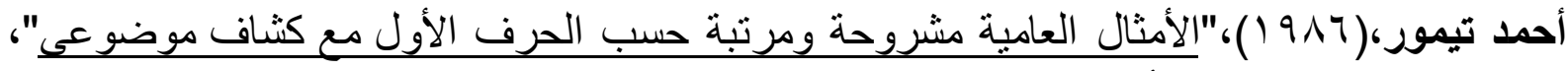

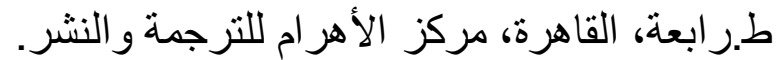

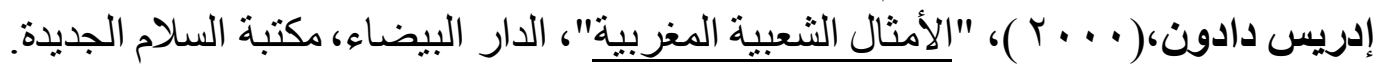

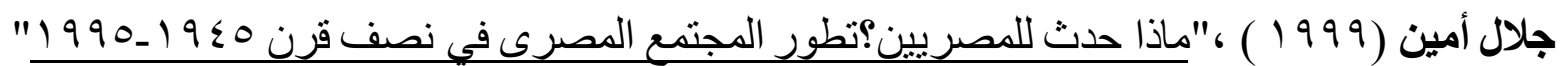
كسلسلة مكتبة الأسرة ،القاهرة ،الهيئة المصرية العامة العادة للكتاب.

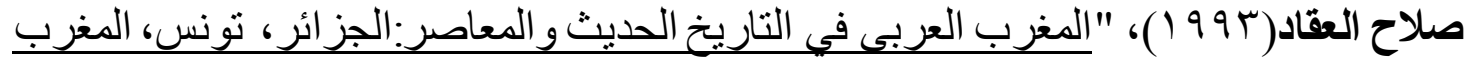

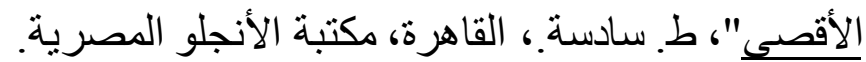

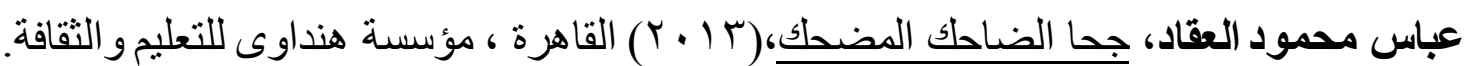

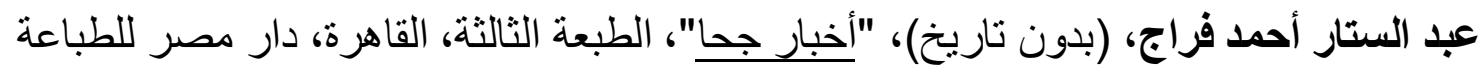

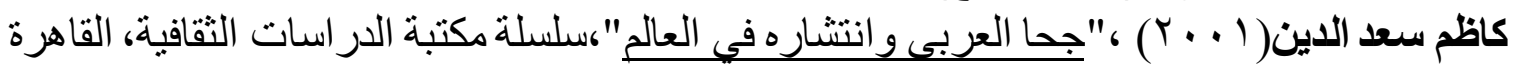

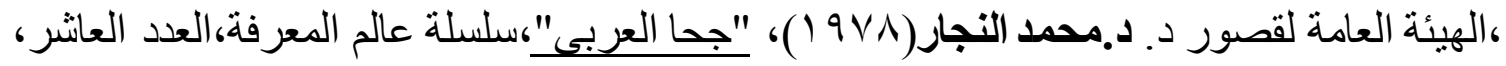
الكويت ،المجلس الوطنى للثقافة و الفنون و اللآداب الثقافة. 